\title{
Is gout a risk equivalent to diabetes for stroke and myocardial infarction? A retrospective claims database study
}

Jasvinder A. Singh ${ }^{1,2,34^{*}}$, Rekha Ramachandaran', Shaohua Yu², Shuo Yang ${ }^{2}$, Fenglong Xie², Huifeng Yun³, Jie Zhang ${ }^{3}$ and Jeffrey R. Curtis 2,3

\begin{abstract}
Background: Gout is a risk factor for cardiovascular disease, but associations with specific cardiovascular outcomes, myocardial infarction (MI), and stroke are unclear. Our objective in the present study was to assess whether gout is as strong a risk factor as diabetes mellitus (DM) for incident $\mathrm{Ml}$ and incident stroke.

Methods: In this retrospective study, we used U.S. claims data from 2007 to 2010 that included a mix of private and public health plans. Four mutually exclusive cohorts were identified: (1) DM only, (2) gout only, (3) gout and DM, and (4) neither gout nor DM. Outcomes were acute Ml or stroke with hospitalization. We compared the age- and sexspecific rates of incident $\mathrm{Ml}$ and stroke across the four cohorts and assessed multivariable-adjusted HRs.
\end{abstract}

Results: In this study, 232,592 patients had DM, 71,755 had gout, 23,261 had both, and 1,010,893 had neither. The incidence of acute Ml was lowest in patients with neither gout nor DM, followed by patients with gout alone, DM alone, and both. Among men $>80$ years of age, the respective rates/1000 person-years were 14.6, 25.4, 27.7, and 37.4. Similar trends were noted for stroke and in women. Compared with DM only, gout was associated with a significantly lower adjusted HR of incident MI (HR 0.81, 95\% Cl 0.76-0.87) but a similar risk of stroke (HR 1.02, 95\% Cl 0.95-1.10). Compared with patients with DM only, patients with both gout and DM had higher HRs for incident Ml and stroke (respectively, HR 1.35, 95\% Cl 1.25-1.47; HR 1.42, 95\% Cl 1.29-1.56).

Conclusions: Gout is a risk equivalent to DM for incident stroke but not for incident MI. Having both gout and DM confers incremental risk compared with DM alone for both incident $\mathrm{Ml}$ and stroke.

Keywords: Gout, Diabetes, Stroke, Myocardial infarction, Risk equivalent

\section{Background}

Gout is the most common inflammatory arthritis in adults [1]. Gout is a risk factor for cardiovascular disease [2], cardiovascular mortality [3], and all-cause mortality $[3,4]$. However, associations with specific cardiovascular outcomes are unclear. Gout was associated with a higher risk of incident myocardial infarction (MI) by 1.23 - to 1.82-fold in some studies [5-8], but it was not associated with higher risk in others $[9,10]$. The association with

\footnotetext{
* Correspondence: jasvinder.md@gmail.com

${ }^{1}$ Medicine Service, VA Medical Center, 700 South 19th Street, Birmingham, AL 35233, USA

${ }^{2}$ Department of Medicine, University of Alabama at Birmingham School of Medicine, 510 20th Street South, Faculty Office Tower 805B, Birmingham, AL 35294-0022, USA

Full list of author information is available at the end of the article
}

stroke is also unclear. Researchers in a U.K. study found that the risk of incident stroke was 1.71 times higher in patients with gout than in the general population [7]. However, their study included only hospitalized patients, who have more severe gout and/or higher comorbidity burden than the general population, thereby limiting the generalizability of their findings [7]. Other limitations of previous studies were that adjustment for key cardiovascular risk factors was not performed in studies of MI $[5,7]$ and stroke [7], which raises a serious concern for significant confounding. Detailed analyses by age and sex (risk varies by these factors) were performed in one study [7], which did not control for cardiovascular risk factors such as hypertension and hyperlipidemia. 
One mechanism of increased cardiovascular risk with gout may be due to hyperuricemia, a central abnormality in gout. Authors of systematic reviews of observational studies have found hyperuricemia by itself (even in the absence of gout) to be associated with a 1.47 times higher risk of stroke [11] and a 1.34 times higher risk of coronary heart disease (CHD) [12]. Hyperuricemia and associated mechanisms may partially mediate increased cardiovascular risk in patients with gout [6,11-14].

Diabetes also is a well-known risk factor for cardiovascular disease. The National Cholesterol Education Program Expert Panel on Detection, Evaluation, and Treatment of High Blood Cholesterol in Adults (Adult Treatment Panel III) labeled diabetes as a cardiovascular risk equivalent, such that lipid management recommendations are similar for patients with diabetes and patients with CHD [15]. Despite being known as a cardiovascular risk factor, it is not known whether gout is a cardiovascular risk equivalent [16] similar to diabetes. Therefore, the objectives of the present study were (1) to assess whether gout is a cardiovascular risk equivalent for incident MI and stroke similar to diabetes, (2) to estimate the increased cardiovascular risk with gout for women and men in various age groups, and (3) to examine if gout is additive to diabetes for cardiovascular risk.

\section{Methods}

We report study methods and results as recommended by the Strengthening the Reporting of Observational Studies in Epidemiology (STROBE) statement [17]. The institutional review board at the University of Alabama at Birmingham approved the study.

\section{Study cohort and participants}

We conducted a retrospective cohort study using the Multi-Payer Claims Database (MPCD) for 2007-2010. This database linked health plan data from national commercial and governmental insurance plans, representing beneficiaries with United Healthcare, Medicare, and/or Medicaid coverage during 2007-2010. The MPCD contains patients' demographic and insurance coverage information from enrollment files, claims for healthcare (inpatient and outpatient) services, and prescription medications. Participants were eligible if they $\mathrm{had} \geq 12$ months of continuous medical and pharmacy coverage to ensure a complete claims history (baseline period). Eligible patients were categorized into four mutually exclusive cohorts: (1) diabetes only, (2) gout only, (3) gout and diabetes, and (4) neither gout nor diabetes. There were no age restrictions on the dataset.

\section{Exposure of interest}

The study exposure of interest was a diagnosis of gout, ascertained by the presence of International Classification of Diseases, Ninth Revision, Clinical Modification (ICD-9-CM) code 274.xx for gout, during an inpatient visit or at two outpatient visits with a physician encounter $\geq 7$ days apart, which has been shown to have high positive predictive value of $>90 \%$ [18]. Diabetes was ascertained by the presence of two ICD-9-CM codes of 250.xx $\geq 7$ days but $\leq 365$ days apart and the use of a diabetes-specific medication, including insulin preparations, biguanide (metformin), thiazolidinediones (pioglitazone, rosiglitazone), sulfonylureas (first generation: tolbutamide, tolazamide, chlorpropamide, acetohexamide; second generation: glipizide, glyburide, glimepiride), sulfonylurea secretagogues (nateglinide, repaglinide), $\alpha$-glucosidase inhibitors (acarbose, miglitol), injectable incretin mimetics (exenatide, liraglutide, taspoglutide, sitagliptin, saxagliptin, linagliptin), injectable amylin analogue (pramlintide), glucagon/dextrose, and drug combinations (e.g., glipizide/metformin) [19], a validated definition with high positive predictive value $>90 \%$ [20-22]. An individual was considered to have diabetes (diabetes cohort-eligible) the day he or she satisfied all these criteria. The index disease when a patient was considered to have diabetes and gout was the more recent of the two dates when a person became gout- and/or diabetes-eligible. Study follow-up started when the later of the following two conditions was met: disease eligibility (gout and diabetes) or 12 months of continuous coverage. Study follow-up ended when either of the two outcomes (described below) occurred; when the patient lost medical or pharmacy coverage; when the patient died; or on December 31, 2010.

\section{Study outcomes}

The study outcomes were the incident hospitalized MI and the incident hospitalized stroke. Incident MI and incident stroke were defined as at least one inpatient claim with hospital discharge with ICD-9-CM codes for acute MI or stroke, respectively (see Additional file 1: Appendix 1 for more detail), and at least one night of inpatient stay, unless the patient died during the index hospitalization. These approaches of identifying patients with incident MI and stroke are valid with a high positive predictive value $>90 \%$ [22-24]. We excluded patients with any of the following conditions in the baseline period of 12 months prior to the start of follow-up (diagnoses of gout and diabetes and 12 months of continuous coverage met) to ensure that we were capturing incident $\mathrm{MI}$ and incident stroke: $\mathrm{MI}$ (410.xx or 412.xx), stroke (430-438) and heart disease (410-414, 428.xx, and 429.2x).

\section{Covariates and confounders}

Study covariates included demographics (age, sex, race/ ethnicity); cardiovascular risk factors other than diabetes 
(hypertension, hyperlipidemia); and other medical comorbidities, including chronic obstructive pulmonary disease (COPD), peripheral vascular disease (PVD), renal failure, and autoimmune diseases selected on the basis of their potential association with the outcome or common occurrence in the population of interest (see Additional file 1: Appendix 1 for more detail). Comorbidities and risk factors were defined on the basis of the presence of ICD-9CM codes for each condition. For hyperlipidemia, we considered patients with either an ICD-9-CM code for hyperlipidemia or the use of a statin drug, because hyperlipidemia is an often undercoded diagnosis [25]. Age was categorized in 5-year age groups, and race/ethnicity was categorized as Asian, Hispanic, black, white, other, or missing. Other variables were categorized as follows: hypertension (yes/no), hyperlipidemia (yes/no), PVD (yes/ no), COPD (yes/no), chronic kidney disease (yes/no), and autoimmune disease (yes/no). Smoking status, fasting lipid levels, and blood pressure readings (in millimeters of mercury) were not available in this database.

\section{Statistical analyses}

We calculated summary statistics as proportions (in percent). We calculated rates of incident MI or incident stroke per 1000 person-years of exposure. We used multivariable-adjusted Cox regression analyses to assess the $\mathrm{HR}$ of incident $\mathrm{MI}$ and incident stroke. We performed unadjusted regression analyses to obtain crude estimates. Age-adjusted and multivariable-adjusted analyses were performed next to account for age (the most significant cardiovascular risk factor) vs. several other cardiovascular risk factors (hypertension, hyperlipidemia) and common comorbidity confounders (COPD). We performed statistical analyses using SAS version 9.3 software (SAS Institute, Cary, NC, USA). The proportional hazards assumption holds true for the analyses reported. A $p$ value $<0.05$ was considered significant. We performed sensitivity analyses by additionally adjusting for the following factors that can potentially impact the risk of $\mathrm{MI} /$ stroke: (1) nonsteroidal anti-inflammatory drugs (NSAIDs; associated increased risk) and medications used for the treatment of heart disease (risk reduction); and (2) autoimmune diseases, owing to associated chronic inflammation.

\section{Results}

\section{Cohort characteristics}

The overall cohort consisted of individuals contributing $1,338,501$ observed study periods. Demographic and clinical characteristics of the four cohorts (diabetes only, gout only, both diabetes and gout, neither diabetes nor gout) are shown in Table 1. Patients with gout, diabetes, or both were slightly older, more likely to be men, and had higher rates of comorbidities than the cohort with neither condition (Table 1). Patients with diabetes only had a higher rate of comorbidities than those without either condition and a lower rate than patients with both gout and diabetes (Table 1). Characteristics of patients with and without incident MI as well as with and without incident stroke are shown in Table 2. Those with incident MI or incident stroke were older, more likely to be white, and more likely to have any of the comorbidities of interest than patients without incident MI or incident stroke (Table 2). The median (25th-75th percentile) follow-up was 427 days (245-988).

\section{Unadjusted incidence of $\mathrm{MI}$ or stroke by age and sex in four cohorts}

Data for the unadjusted incidence of MI (Table 3) and stroke per 1000 patient-years (Table 4) are provided by sex within each age group (see also Fig. 1). Unadjusted incidence rates for MI in patients with only gout were similar to or slightly lower than in patients with only diabetes and much higher for patients with both gout and diabetes (Table 3 and Fig. 1a and b). Unadjusted incidence rates for stroke in patients with only gout were similar to or slightly higher than in patients with only diabetes (Table 4 and Fig. 1c and d). Rates for MI and stroke increased with age in all four cohorts (Fig. 1).

\section{Gout vs. diabetes as a risk of incident MI or incident stroke}

In age-adjusted analyses, compared with diabetes alone, patients with gout alone had a lower risk of MI but a similar risk of stroke, with respective HRs of 0.87 (95\% CI 0.81-0.92) and 1.06 (95\% CI 0.99-1.14) (Table 5).

In multivariable-adjusted analyses, compared with patients with only diabetes, those with only gout had significantly lower HRs of incident MI (HR 0.81, 95\% CI 0.76-0.87) but a similar HR of incident stroke (HR 1.02, 95\% CI 0.95-1.10) (Table 5). Compared with patients with only diabetes, patients with both gout and diabetes had significantly higher risk for incident MI (HR 1.35, 95\% CI 1.25-1.47) and of incident stroke (HR 1.42, 95\% CI 1.29-1.56), and those with neither disease had significantly lower HRs of incident MI and incident stroke (Table 5). Conversely, compared with patients without diabetes and gout, higher HRs of incident MI were significantly higher: gout (HR 1.53, 95\% CI 1.44-1.63) and both diabetes and gout (HR 2.55, 95\% CI 2.35-2.77). Details of the full model, including all covariates, are shown in Additional file 1: Appendix 2. Other significant factors associated with higher HRs for incident MI were older age, male sex, nonwhite race/ethnicity, hyperlipidemia, COPD, and renal disease. Factors significantly associated with incident stroke were similar, except that sex was not significantly associated.

Sensitivity analyses additionally adjusted for use of NSAIDs and antihypertensive drugs revealed minimal 
Table 1 Patient characteristics

\begin{tabular}{|c|c|c|c|c|}
\hline & DM only $(n=232,592)$ & Gout only $(n=71,755)$ & DM and gout $(n=23,261)$ & Neither DM nor gout $(n=1,010,893)$ \\
\hline \multirow[t]{2}{*}{ Male sex } & 97,041 & 48,305 & 13,192 & 395,608 \\
\hline & $(41.7 \%)$ & $(67.3 \%)$ & $(56.7 \%)$ & (39.1\%) \\
\hline \multicolumn{5}{|l|}{ Age group, years } \\
\hline \multirow[t]{2}{*}{$\leq 50$} & 41,844 & 7910 & 1616 & 395,186 \\
\hline & $(18.0 \%)$ & $(11.0 \%)$ & $(7.0 \%)$ & (39.1\%) \\
\hline \multirow[t]{2}{*}{$51-60$} & 43,982 & 7589 & 3057 & 125,502 \\
\hline & $(18.9 \%)$ & $(10.6 \%)$ & $(13.1 \%)$ & $(12.4 \%)$ \\
\hline \multirow[t]{2}{*}{$61-65$} & 30,762 & 6268 & 2611 & 78,983 \\
\hline & $(13.2 \%)$ & $(8.7 \%)$ & $(11.2 \%)$ & $(7.8 \%)$ \\
\hline \multirow[t]{2}{*}{$66-70$} & 40,974 & 14,044 & 5066 & 121,976 \\
\hline & $(17.6 \%)$ & $(19.6 \%)$ & $(21.8 \%)$ & $(12.1 \%)$ \\
\hline \multirow[t]{2}{*}{$71-75$} & 30,601 & 12,055 & 4392 & 97,567 \\
\hline & $(13.2 \%)$ & $(16.8 \%)$ & $(18.9 \%)$ & $(9.7 \%)$ \\
\hline \multirow[t]{2}{*}{$76-80$} & 22,807 & 10,386 & 3227 & 82,298 \\
\hline & $(9.8 \%)$ & $(14.5 \%)$ & $(13.9 \%)$ & (8.1\%) \\
\hline \multirow[t]{2}{*}{$>80$} & 21,620 & 13,501 & 3292 & 108,785 \\
\hline & $(9.3 \%)$ & $(18.8 \%)$ & $(14.2 \%)$ & $(10.8 \%)$ \\
\hline \multicolumn{5}{|l|}{ Race } \\
\hline \multirow[t]{2}{*}{ Asian } & 6533 & 3270 & 970 & 24,744 \\
\hline & $(2.8 \%)$ & $(4.6 \%)$ & $(4.2 \%)$ & $(2.5 \%)$ \\
\hline \multirow[t]{2}{*}{ Black } & 34,551 & 10,358 & 4548 & 118,867 \\
\hline & $(14.9 \%)$ & $(14.4 \%)$ & $(19.6 \%)$ & (11.8\%) \\
\hline \multirow[t]{2}{*}{ Hispanic } & 14,100 & 1938 & 557 & 70,382 \\
\hline & $(6.1 \%)$ & $(2.7 \%)$ & $(2.4 \%)$ & $(7.0 \%)$ \\
\hline \multirow[t]{2}{*}{ Other } & 4199 & 934 & 470 & 11,254 \\
\hline & $(1.8 \%)$ & $(1.3 \%)$ & $(2.0 \%)$ & $(1.1 \%)$ \\
\hline \multirow[t]{2}{*}{ Missing } & 27,372 & 6418 & 1258 & 222,928 \\
\hline & $(11.8 \%)$ & (8.9\%) & $(5.4 \%)$ & (22.1\%) \\
\hline \multirow[t]{2}{*}{ White } & 145,837 & 48,837 & 15,458 & 562,718 \\
\hline & $(62.7 \%)$ & $(68.1 \%)$ & $(66.5 \%)$ & (55.7\%) \\
\hline \multicolumn{5}{|l|}{ Comorbidities } \\
\hline \multirow[t]{2}{*}{ Hypertension } & 168,155 & 47,446 & 19,556 & 273,828 \\
\hline & $(72.3 \%)$ & $(66.1 \%)$ & $(84.1 \%)$ & (27.1\%) \\
\hline \multirow[t]{2}{*}{ COPD } & 20,835 & 5837 & 2376 & 50,610 \\
\hline & $(9.0 \%)$ & $(8.1 \%)$ & $(10.2 \%)$ & $(5.0 \%)$ \\
\hline \multirow[t]{2}{*}{ Chronic kidney disease } & 20,078 & 9366 & 5641 & 22,791 \\
\hline & $(8.6 \%)$ & $(13.1 \%)$ & $(24.3 \%)$ & $(2.3 \%)$ \\
\hline \multirow[t]{2}{*}{ Peripheral vascular disease } & 14,362 & 3850 & 1997 & 22,078 \\
\hline & $(6.2 \%)$ & $(5.4 \%)$ & $(8.6 \%)$ & $(2.2 \%)$ \\
\hline \multirow[t]{2}{*}{ Hyperlipidemia } & 168,964 & 36,296 & 18,066 & 262,591 \\
\hline & $(72.6 \%)$ & $(50.6 \%)$ & $(77.7 \%)$ & (26.0\%) \\
\hline
\end{tabular}

Abbreviations: DM Diabetes mellitus, COPD Chronic obstructive pulmonary disease

Hyperlipidemia is defined as a presence of a diagnostic code for hypercholesterolemia or statin use. Diagnostic codes for comorbidities are provided in Additional file 1: Appendix 1 
Table 2 Characteristics of patients with incident myocardial infarction or incident stroke

\begin{tabular}{|c|c|c|c|c|}
\hline & $\begin{array}{l}\text { No Ml } \\
(n=1,325,037)\end{array}$ & $\begin{array}{l}\text { Incident Ml } \\
(n=13,464)\end{array}$ & $\begin{array}{l}\text { No stroke } \\
(n=1,328,015)\end{array}$ & $\begin{array}{l}\text { Incident stroke } \\
(n=10,486)\end{array}$ \\
\hline \multirow[t]{2}{*}{ Male sex } & 548,447 & 5699 & 550,342 & 3804 \\
\hline & $(41.4 \%)$ & $(42.3 \%)$ & $(41.4 \%)$ & (36.3\%) \\
\hline \multicolumn{5}{|l|}{ Age group, years } \\
\hline \multirow[t]{2}{*}{$\leq 50$} & 445,916 & 640 & 446,085 & 471 \\
\hline & (33.7\%) & $(4.8 \%)$ & (33.6\%) & $(4.5 \%)$ \\
\hline \multirow[t]{2}{*}{$51-60$} & 179,005 & 1125 & 179,348 & 782 \\
\hline & $(13.5 \%)$ & $(8.4 \%)$ & $(13.5 \%)$ & $(7.5 \%)$ \\
\hline \multirow[t]{2}{*}{$61-65$} & 117,717 & 907 & 117,917 & 707 \\
\hline & $(8.9 \%)$ & $(6.7 \%)$ & $(8.9 \%)$ & $(6.7 \%)$ \\
\hline \multirow[t]{2}{*}{$66-70$} & 179,931 & 2129 & 180,550 & 1510 \\
\hline & $(13.6 \%)$ & $(15.8 \%)$ & $(13.6 \%)$ & $(14.4 \%)$ \\
\hline \multirow[t]{2}{*}{$71-75$} & 142,369 & 2246 & 142,889 & 1726 \\
\hline & $(10.7 \%)$ & $(16.7 \%)$ & $(10.8 \%)$ & $(16.5 \%)$ \\
\hline \multirow[t]{2}{*}{$76-80$} & 116,375 & 2343 & 116,781 & 1937 \\
\hline & $(8.8 \%)$ & $(17.4 \%)$ & $(8.8 \%)$ & $(18.5 \%)$ \\
\hline \multirow[t]{2}{*}{$>80$} & 143,125 & 4073 & 143,846 & 3352 \\
\hline & $(10.8 \%)$ & $(30.3 \%)$ & $(10.8 \%)$ & (32.0\%) \\
\hline \multicolumn{5}{|l|}{ Race } \\
\hline \multirow[t]{2}{*}{ Asian } & 35,218 & 299 & 35,269 & 248 \\
\hline & $(2.7 \%)$ & $(2.2 \%)$ & $(2.7 \%)$ & $(2.4 \%)$ \\
\hline \multirow[t]{2}{*}{ Black } & 166,744 & 1580 & 166,617 & 1707 \\
\hline & $(12.6 \%)$ & $(11.7 \%)$ & $(12.6 \%)$ & $(16.3 \%)$ \\
\hline \multirow[t]{2}{*}{ Hispanic } & 86,542 & 435 & 86,607 & 370 \\
\hline & $(6.5 \%)$ & $(3.2 \%)$ & $(6.5 \%)$ & (3.5\%) \\
\hline \multirow[t]{2}{*}{ Other } & 16,688 & 169 & 16,699 & 158 \\
\hline & $(1.3 \%)$ & $(1.3 \%)$ & $(1.3 \%)$ & $(1.5 \%)$ \\
\hline \multirow[t]{2}{*}{ Missing } & 257,540 & 436 & 257,627 & 349 \\
\hline & $(19.4 \%)$ & $(3.2 \%)$ & $(19.4 \%)$ & (3.3\%) \\
\hline \multirow[t]{2}{*}{ White } & 762,305 & 10,545 & 765,196 & 7654 \\
\hline & $(57.5 \%)$ & $(78.3 \%)$ & $(57.6 \%)$ & $(73.0 \%)$ \\
\hline \multicolumn{5}{|l|}{ Comorbidities } \\
\hline \multirow[t]{2}{*}{ Hypertension } & 500,421 & 8564 & 502,233 & 6752 \\
\hline & $(37.8 \%)$ & $(63.6 \%)$ & $(37.8 \%)$ & $(64.4 \%)$ \\
\hline \multirow[t]{2}{*}{ COPD } & 77,833 & 1825 & 78,533 & 1125 \\
\hline & $(5.9 \%)$ & $(13.6 \%)$ & $(5.9 \%)$ & $(10.7 \%)$ \\
\hline \multirow[t]{2}{*}{ Chronic kidney disease } & 56,131 & 1745 & 56,741 & 1135 \\
\hline & $(4.2 \%)$ & $(13.0 \%)$ & $(4.3 \%)$ & $(10.8 \%)$ \\
\hline \multirow[t]{2}{*}{ Peripheral vascular disease } & 40,919 & 1368 & 41,446 & 841 \\
\hline & $(3.1 \%)$ & $(10.2 \%)$ & (3.1\%) & $(8.0 \%)$ \\
\hline \multirow[t]{2}{*}{ Hyperlipidemia } & 478,544 & 7373 & 480,711 & 5206 \\
\hline & $(36.1 \%)$ & $(54.8 \%)$ & $(36.2 \%)$ & $(49.7 \%)$ \\
\hline
\end{tabular}

Abbreviations: MI Myocardial infarction, COPD Chronic obstructive pulmonary disease

Data were analyzed after removing the patients with baseline International Classification of Diseases, Ninth Revision, Clinical Modification, codes of 410,412 , 430-438, 428.xx, and 429.2x for Ml and stroke 
Table 3 Unadjusted rates of incident myocardial infarction by age and sex based on the presence/absence of diabetes and gout

\begin{tabular}{|c|c|c|c|c|c|c|}
\hline \multirow{2}{*}{$\begin{array}{l}\text { Group } \\
\text { Age groups } \\
\text { (years) }\end{array}$} & \multicolumn{3}{|l|}{ Females } & \multicolumn{3}{|l|}{ Males } \\
\hline & No. of Ml events & Person-years & $\begin{array}{l}\text { Incidence rate } \\
\text { (/1000 PY) }\end{array}$ & No. of Ml events & Person-years & $\begin{array}{l}\text { Incidence rate } \\
\text { (/1000 PY) }\end{array}$ \\
\hline \multicolumn{7}{|c|}{ Diabetes and gout } \\
\hline$\leq 50$ & 5 & 519.1 & 9.63 & 16 & 1665.0 & 9.61 \\
\hline $51-60$ & 22 & 1463.6 & 15.03 & 45 & 2642.8 & 17.03 \\
\hline $61-65$ & 14 & 1376.8 & 10.17 & 32 & 2103.6 & 15.21 \\
\hline $66-70$ & 51 & 3067.2 & 16.63 & 67 & 4353.8 & 15.39 \\
\hline $71-75$ & 65 & 2992.6 & 21.72 & 83 & 3507.9 & 23.66 \\
\hline $76-80$ & 51 & 2531.1 & 20.15 & 66 & 2303.4 & 28.65 \\
\hline$>80$ & 106 & 2688.5 & 39.43 & 64 & 1709.0 & 37.45 \\
\hline \multicolumn{7}{|c|}{ Diabetes only, no gout } \\
\hline$\leq 50$ & 113 & $32,057.2$ & 3.52 & 125 & $27,800.2$ & 4.50 \\
\hline $51-60$ & 269 & $38,836.8$ & 6.93 & 229 & $29,492.3$ & 7.76 \\
\hline $61-65$ & 206 & $27,596.4$ & 7.46 & 181 & $20,494.1$ & 8.83 \\
\hline $66-70$ & 439 & $45,356.6$ & 9.68 & 360 & $33,703.8$ & 10.68 \\
\hline $71-75$ & 463 & $37,735.6$ & 12.27 & 339 & $23,503.7$ & 14.42 \\
\hline $76-80$ & 445 & $30,209.4$ & 14.73 & 293 & $15,681.8$ & 18.68 \\
\hline$>80$ & 696 & $29,606.4$ & 23.51 & 317 & $11,422.9$ & 27.75 \\
\hline \multicolumn{7}{|c|}{ Gout only, no diabetes } \\
\hline$\leq 50$ & 6 & 1177.0 & 5.10 & 36 & 8691.4 & 4.14 \\
\hline $51-60$ & 9 & 2011.3 & 4.47 & 46 & 7845.4 & 5.86 \\
\hline $61-65$ & 8 & 2153.1 & 3.72 & 37 & 5882.7 & 6.29 \\
\hline $66-70$ & 43 & 5861.1 & 7.34 & 132 & $14,896.7$ & 8.86 \\
\hline $71-75$ & 53 & 6308.6 & 8.40 & 144 & $12,016.8$ & 11.98 \\
\hline $76-80$ & 70 & 6510.9 & 10.75 & 156 & 9253.9 & 16.86 \\
\hline$>80$ & 247 & $10,232.1$ & 24.14 & 221 & 8705.4 & 25.39 \\
\hline \multicolumn{7}{|c|}{ No gout or diabetes } \\
\hline$\leq 50$ & 178 & $293,997.0$ & 0.61 & 161 & $222,722.0$ & 0.72 \\
\hline $51-60$ & 277 & $121,761.0$ & 2.27 & 228 & $76,284.1$ & 2.99 \\
\hline $61-65$ & 238 & $76,628.8$ & 3.11 & 191 & $43,196.1$ & 4.42 \\
\hline $66-70$ & 528 & $140,311.0$ & 3.76 & 508 & $80,566.3$ & 6.31 \\
\hline $71-75$ & 614 & $119,092.0$ & 5.16 & 485 & $62,948.0$ & 7.70 \\
\hline $76-80$ & 810 & $105,226.0$ & 7.70 & 452 & $48,350.6$ & 9.35 \\
\hline$>80$ & 1738 & $144,580.0$ & 12.02 & 684 & $46,825.3$ & 14.61 \\
\hline
\end{tabular}

MI Myocardial infarction, PY Person-years

*Baseline International Classification of Diseases, Ninth Revision, Clinical Modification, codes of 410, 412, 430-438, 428.xx, and 429.2X were removed to find incident $\mathrm{MI}$ rates

change in ORs/HRs and no change in the interpretation of study results (see Additional file 1: Appendix 3 for more detail). Sensitivity analyses additionally adjusted for immune diseases, another potential risk factor for MI and stroke owing to associated chronic inflammation, led to minimal attenuation of HR but no change in $p$ value or interpretation (data not shown, available from author on request).

\section{Discussion}

Using a large cohort of patients who had gout, diabetes, both, or neither, we investigated whether gout was a risk equivalent for incident MI and stroke, similar to diabetes. We used a systematic approach to address this by analyzing a large U.S. sample and comparing patients with diabetes and gout, patients with only gout, and those with only diabetes. On one hand, our findings 
Table 4 Unadjusted rates of incident* stroke by age and sex based on the presence/absence of diabetes and gout

\begin{tabular}{|c|c|c|c|c|c|c|}
\hline \multirow{2}{*}{$\begin{array}{l}\text { Group } \\
\text { Age groups } \\
\text { (years) }\end{array}$} & \multicolumn{3}{|l|}{ Females } & \multicolumn{3}{|l|}{ Males } \\
\hline & No. of stroke events & Person-years & $\begin{array}{l}\text { Incidence rate } \\
\text { (/1000 PY) }\end{array}$ & No. of stroke events & Person-years & $\begin{array}{l}\text { Incidence rate } \\
\text { (/1000 PY) }\end{array}$ \\
\hline \multicolumn{7}{|c|}{ Diabetes and gout } \\
\hline$\leq 50$ & 2 & 507.1 & 3.94 & 5 & 1681.4 & 2.97 \\
\hline $51-60$ & 10 & 1444.4 & 6.92 & 25 & 2629.4 & 9.51 \\
\hline $61-65$ & 17 & 1329.1 & 12.79 & 21 & 2094.0 & 10.03 \\
\hline $66-70$ & 31 & 2960.7 & 10.47 & 47 & 4275.3 & 10.99 \\
\hline $71-75$ & 64 & 2828.7 & 22.63 & 58 & 3392.6 & 17.10 \\
\hline $76-80$ & 50 & 2412.2 & 20.73 & 40 & 2195.6 & 18.22 \\
\hline$>80$ & 82 & 2543.8 & 32.23 & 44 & 1633.0 & 26.94 \\
\hline \multicolumn{7}{|c|}{ Diabetes only, no gout } \\
\hline$\leq 50$ & 95 & $31,642.3$ & 3.00 & 66 & $27,586.3$ & 2.39 \\
\hline $51-60$ & 230 & $37,988.2$ & 6.05 & 123 & $29,100.9$ & 4.23 \\
\hline $61-65$ & 168 & $26,836.9$ & 6.26 & 142 & $20,078.1$ & 7.07 \\
\hline $66-70$ & 295 & $43,522.1$ & 6.78 & 235 & $32,805.9$ & 7.16 \\
\hline $71-75$ & 311 & $35,848.3$ & 8.68 & 230 & $22,574.2$ & 10.19 \\
\hline $76-80$ & 348 & 28,436 & 12.24 & 189 & $14,887.7$ & 12.70 \\
\hline$>80$ & 578 & $27,758.8$ & 20.82 & 171 & $10,827.8$ & 15.79 \\
\hline \multicolumn{7}{|c|}{ Gout only, no diabetes } \\
\hline$\leq 50$ & 4 & 1175.3 & 3.40 & 14 & 8647.9 & 1.62 \\
\hline $51-60$ & 9 & 1964.2 & 4.58 & 29 & 7795.3 & 3.72 \\
\hline $61-65$ & 14 & 2086.0 & 6.71 & 34 & 5772.2 & 5.89 \\
\hline $66-70$ & 44 & 5705.1 & 7.71 & 110 & $14,624.7$ & 7.52 \\
\hline $71-75$ & 71 & 6019.3 & 11.80 & 117 & $11,595.8$ & 10.09 \\
\hline $76-80$ & 85 & 6172.5 & 13.77 & 132 & 8866.4 & 14.89 \\
\hline$>80$ & 247 & 9655.8 & 25.58 & 168 & 8324.6 & 20.18 \\
\hline \multicolumn{7}{|c|}{ No gout or diabetes } \\
\hline$\leq 50$ & 181 & $292,332.0$ & 0.62 & 104 & $222,180.0$ & 0.47 \\
\hline $51-60$ & 235 & $120,045.0$ & 1.96 & 121 & $75,642.6$ & 1.60 \\
\hline $61-65$ & 197 & $75,185.7$ & 2.62 & 114 & $42,652.2$ & 2.67 \\
\hline $66-70$ & 443 & $136,150.0$ & 3.25 & 303 & $78,992.1$ & 3.84 \\
\hline $71-75$ & 549 & $114,537.0$ & 4.79 & 326 & $61,136.1$ & 5.33 \\
\hline $76-80$ & 737 & $100,637.0$ & 7.32 & 356 & $46,603.7$ & 7.64 \\
\hline$>80$ & 1581 & $138,276.0$ & 11.43 & 479 & $44,873.5$ & 10.67 \\
\hline
\end{tabular}

PY Person-years

*Baseline International Classification of Diseases, Ninth Revision, Clinical Modification, codes of 410, 412, 430-438, 428.xx, and 429.2X were removed to find incident stoke rates

indicate that gout was a cerebrovascular risk factor equivalent, similar to diabetes, for stroke. On the other hand, gout was not a cardiovascular risk factor equivalent for incident MI, unlike diabetes. Patients with both gout and diabetes had a 1.4 times multivariable-adjusted risk of incident MI and stroke for each, compared with only diabetes, demonstrating that gout was additive to diabetes in further increasing the risk of stroke and MI, above and beyond the increased risk by diabetes. These novel findings merit further discussion.

To our knowledge, this is the first large observational study to examine gout as a cerebrovascular risk equivalent for stroke equivalent to diabetes. The adjusted HRs for incident stroke were comparable between patients with gout alone and patients with diabetes alone, showing that gout is a cerebrovascular risk equivalent to 


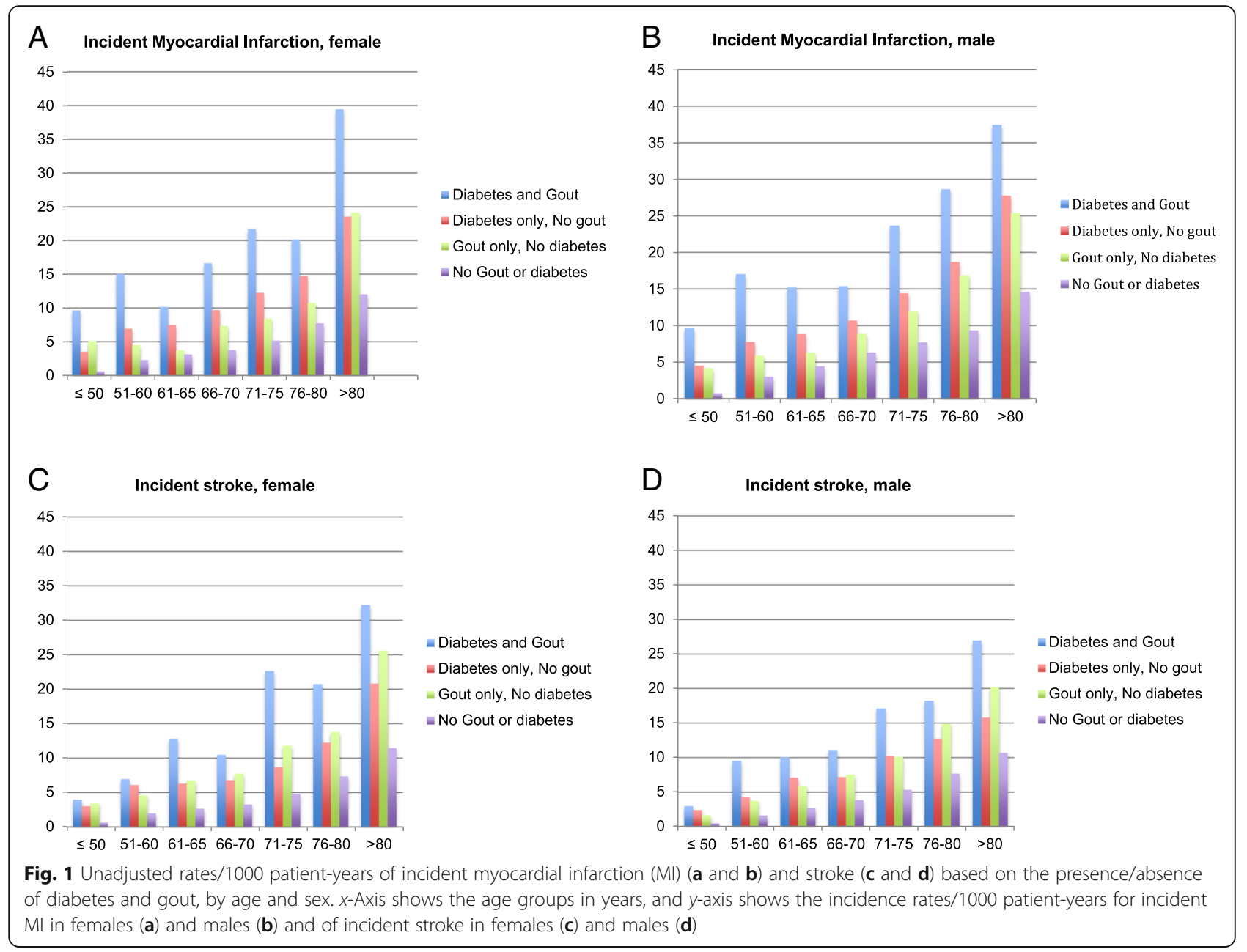

Table 5 Unadjusted, age-adjusted, and multivariable-adjusted HR of incident myocardial infarction or incident stroke

\begin{tabular}{|c|c|c|c|c|c|c|}
\hline & \multicolumn{3}{|c|}{$\begin{array}{l}\text { Incident MI HR }(95 \% \mathrm{Cl}) \\
p \text { value }\end{array}$} & \multicolumn{3}{|c|}{$\begin{array}{l}\text { Incident stroke HR }(95 \% \mathrm{Cl}) \\
p \text { value }\end{array}$} \\
\hline & Unadjusted & Age-adjusted & Multivariable-adjusted $^{a}$ & Unadjusted & Age-adjusted & Multivariable-adjusted $^{2}$ \\
\hline \multirow[t]{2}{*}{ Gout and diabetes } & $1.89(1.75-2.05)$ & $1.61(1.49-1.75)$ & $1.35(1.25-1.47)$ & $1.91(1.74-2.10)$ & $1.61(1.46-1.77)$ & $1.42(1.29-1.56)$ \\
\hline & $p<0.0001$ & $p<0.0001$ & $p<0.0001$ & $p<0.0001$ & $p<0.0001$ & $p<0.0001$ \\
\hline \multirow[t]{2}{*}{ Gout, no diabetes } & $1.08(1.01-1.15)$ & $0.87(0.81-0.92)$ & $0.81(0.76-0.87)$ & $1.35(1.26-1.45)$ & $1.06(0.99-1.14)$ & $1.02(0.95-1.10)$ \\
\hline & $p=0.0187$ & $p<0.0001$ & $p<0.0001$ & $p<0.0001$ & $p=0.1005$ & $p=0.5723$ \\
\hline \multirow[t]{2}{*}{ No diabetes, no gout } & $0.41(0.39-0.42)$ & $0.45(0.43-0.46)$ & $0.53(0.51-0.55)$ & $0.45(0.43-0.47)$ & $0.50(0.48-0.52)$ & $0.57(0.54-0.60)$ \\
\hline & $p<0.0001$ & $p<0.0001$ & $p<0.0001$ & $p<0.0001$ & $p<0.0001$ & $p<0.0001$ \\
\hline Diabetes, no gout & Reference & Reference & Reference & Reference & Reference & Reference \\
\hline
\end{tabular}

MI Myocardial infarction

A nonsignificant HR in age- and multivariable-adjusted models denotes that, with patients with diabetes as the reference category, the HR of incident stroke was similar; in other words, gout is a cerebrovascular risk factor equivalent to diabetes.

Multivariable-adjusted analysis included patients without MI, stroke, and heart disease in the baseline period

${ }^{a}$ Adjusted for age, sex, race, hypertension, hyperlipidemia, chronic obstructive pulmonary disease, chronic kidney disease, and peripheral vascular disease.

Additional adjustment for immune diseases led to minimal attenuation of OR but no change in $p$ value or interpretation 
diabetes. Patients with both diabetes and gout had a 1.4 times multivariable-adjusted HR of incident stroke compared with diabetes alone, further supporting the additive risk of gout to diabetes [15] in patients without underlying coronary artery disease (CAD). However, gout is not a risk equivalent to diabetes for incident MI, an important negative finding in our study. We addressed this important previously raised question of risk equivalency. Not surprisingly, patients with gout and diabetes had higher HRs of MI than those with only diabetes (HR 1.35, 95\% CI 1.25-1.47) and those without gout or diabetes (HR 2.55, 95\% CI 2.35-2.77). Thus, our study confirms previous findings that gout is an independent risk factor for MI $[2-8,26]$ and shows that MI risk with gout was additive to the higher risk noted with diabetes. This finding highlights the difference in being a risk factor vs. a risk equivalent to diabetes.

In a recent analysis not adjusted for important cardiovascular risk factors such as hypertension and diabetes compared with the U.K. general population, people with gout had a 1.7 times higher risk of incident stroke [7]. The study included only hospitalized patients with a diagnosis of gout, which might represent a sicker patient subgroup. Thus, the study population, design, and methodology were not comparable to our study. Observational studies adjusted for cardiovascular risk factors showed that gout was associated with 1.26-1.82 times higher odds of MI [6-8] vs. not associated with incident cardiovascular disease $[9,10]$ vs. associated with MI only in women and not in men [5]. Our study shows that gout was an independent risk factor for incident $\mathrm{MI}$ and of incident stroke in patients without underlying CAD.

There are several proposed mechanisms that can mediate the increased stroke risk in patients with gout, such that gout is a stroke risk equivalent of diabetes. Hyperuricemia, the key underlying abnormality in gout, impairs nitric oxide production $[27,28]$ and activates the renin-angiotensin system [29], leading to endothelial dysfunction [30,31], which also contributes to increased blood pressure [32, 33]. Persistent systemic and vascular inflammation in gout due to hyperuricemia $[34,35]$ and monosodium urate crystals $[36,37]$ may also contribute to atherosclerosis and stimulate prothrombotic activity [38]. Studies show that uric acid stimulates vascular smooth muscle cell proliferation [39-41]. These mechanisms can potentially explain an increased risk of atherosclerosis $[42,43]$ and a resulting increase in the risk of incident stroke with gout, similar to that seen with diabetes (risk equivalent). This difference in gout being a risk equivalent to diabetes for stroke but not for MI may be due to different pathogenic roles of gout and urate crystals in stroke vs. MI. Gout also increases the risk of atrial fibrillation [44, 45], a risk factor for stroke but not MI, which may also increase the difference. Systemic inflammation associated with gout may have a differential effect on the risk of incident MI vs. stroke. In this database study, we did not perform any laboratory assays that could provide more insight into underlying mechanisms of acute MI or stroke. Future prospective studies can address this knowledge gap. Despite a few similar risk factors (sex, age, hypertension, diabetes, smoking, obesity, dyslipidemia), other risk factors for stroke (atrial fibrillation, alcohol use) differ from those for MI (modest alcohol use reduces risk) as well as pathogenesis [46-48].

The clinical implications of these study findings may be significant. Although our findings need confirmation in future studies, our recognition of gout as a cerebrovascular risk equivalent to diabetes implies that primary, secondary, and tertiary prevention of stroke may be needed in patients with gout to reduce this increased risk of stroke. On the basis of these findings, screening and aggressive treatment of risk factors for stroke (hypertension, diabetes, hyperlipidemia) may be warranted in patients with gout. It remains to be seen whether physicians may also consider empiric use of lipid-lowering agents in a subgroup of patients with gout at high risk of cardiovascular events, similar to an approach in diabetes, but this needs further study. Optimal treatment of gout targeting both hyperuricemia and systematic inflammation may also be needed to reduce the risk of stroke in patients with gout. What remains to be seen is to what extent each of these strategies reduces the risk of stroke in patients with gout. Large-sample observational studies and/or randomized trials in high-risk gout patients can answer this question in the future. These study findings indicate that cardiovascular disease prevention and treatment strategies that are in place for patients with diabetes [15] may also be applicable to patients with gout.

Gout has been linked to higher overall mortality in previous studies $[3,4,49]$, including higher cardiovascular mortality $[3,50]$. MI and stroke are major contributors to cardiovascular mortality and to overall mortality. Our study provides further insight and a further rationale for this previous finding by showing that gout is a cerebrovascular risk equivalent of diabetes for stroke, which is associated with high cardiovascular mortality. Authors of a recent review explained the links of uric acid to cardiovascular disease by examining its associations with oxidative stress, inflammation, and immune activation [51].

Several limitations must be considered while interpreting the findings of our study. Smoking status and lipid levels (including high-density lipoprotein) were not available in our database, and therefore we could not adjust for these Framingham risk factors in our analyses. We used a diagnostic code for hypercholesterolemia or the use of statins to capture patients with 
hypercholesterolemia, which may still have led to underdiagnosis owing to some patients not wanting/taking statins for hypercholesterolemia and owing to undercoding. Other potential risk factors for $\mathrm{MI} /$ stroke, including body mass index and alcohol use, were not available in the database; therefore, we were not able to adjust for these factors in the analyses. Serum urate levels were available for a very small proportion of patients, not allowing us to incorporate this in mediation analysis in this database study. Therefore, we could not examine whether the associations were mediated via hyperuricemia and whether the effects differed by the level of serum urate.

Another study limitation was that the study period of 2007-2010 resulted in a short mean follow-up period; however, we had 1.34 million observed study periods, which provided adequate study power. As in most commercial claims datasets, death was not available as a variable, aside from in-hospital mortality, which was not sufficient to run a competing risk analysis. We used prevalent diabetes and gout rather than incident gout or diabetes to have enough cases of outcomes of interest, incident stroke or MI, a key study consideration in this analysis. Researchers in future studies should consider using incident disease instead of prevalent disease. Positive predictive value for gout diagnosis was based on a study done at a Veterans Affairs (VA) medical center [18], and it is possible that coding in the Medicare system may be either more or less accurate than in the VA system. The very high prevalence of hyperlipidemia in patients with diabetes and the high prevalence in other populations (gout, neither) in our study are similar to the reported prevalence in the U.S. general population and in subjects with diabetes $[52,53]$; inclusion of the use of statins in the definition may have increased the sensitivity of the definition. Study strengths include a large sample size and the availability of a cohort with diabetes, allowing comparison to a risk equivalent, robust estimates tested in multiple sensitivity analyses, and use of a population without baseline heart disease.

\section{Conclusions}

We found that, compared with diabetes, gout was a risk equivalent for incident stroke but not a risk equivalent for incident MI. Gout was additive to diabetes in increasing the risk of both incident MI and incident stroke. As an inflammatory disease commonly associated with high comorbidity load (hypertension, diabetes, hyperlipidemia, obesity, renal failure) and systemic inflammation, gout can be effectively treated with inexpensive generic medications. On the basis of our study results, the presence of gout should alert physicians to screen, diagnose, and promptly treat cardiovascular risk factors (hypertension, hyperlipidemia), similar to the approach used in patients with diabetes. The increased risk of stroke may prompt patients and physicians to consider empiric lipid-lowering treatment in at least those patients with gout who have high cardiovascular risk and might be predisposed to poor outcomes. Researchers in future studies need to examine the strategies for such as approach and estimate its effect on stroke and other cardiovascular outcomes in patients with gout.

\section{Additional file}

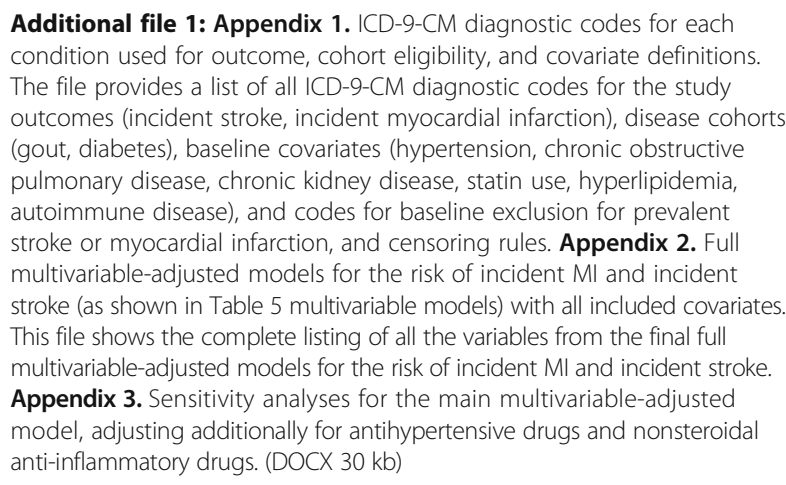

\section{Abbreviations}

CAD: Coronary artery disease; CHD: Coronary heart disease; COPD: Chronic obstructive pulmonary disease; DM: Diabetes mellitus; ICD-9-

CM: International Classification of Diseases, Ninth Revision, Clinical Modification; MI: Myocardial infarction; MPCD: Multi-Payer Claims Database; NSAID: Nonsteroidal anti-inflammatory drug; PVD: Peripheral vascular disease; STROBE: Strengthening the Reporting of Observational Studies in Epidemiology; VA: Veterans Affairs

\section{Acknowledgements}

We thank the Actuarial Research Corporation for providing us with the data.

\section{Funding}

This study was supported by the Actuarial Research Corporation (on behalf of the U.S. Department of Health and Human Services) and the resources and the use of facilities at the Birmingham VA Medical Center (Birmingham, AL, USA). JAS is supported by research grants from the National Institute of Arthritis and Musculoskeletal and Skin Diseases (NIAMS), the National Institute on Aging (NIA), the National Cancer Institute (NCI), the Agency for Healthcare Research and Quality, and the Centers for Education and Research on Therapeutics (CERTs). The views expressed in this article are those of the authors and do not necessarily reflect the position or policy of the Department of Veterans Affairs or the U.S. government.

\section{Availability of data and materials}

We are ready to share the data reported in this article with colleagues after appropriate institutional, ethics, and patient privacy requirements have been met.

\section{Authors' contributions}

JAS and JRC conceived of the idea for the study. JAS developed the initial study protocol. RR performed data programming. RR and SYu performed the data analyses. SYa, FX, HY, and JZ assisted with data programming. All authors reviewed the results and provided feedback. JAS wrote the first draft of the manuscript. All authors critically revised the paper. All authors read and approved the final manuscript. 


\section{Authors' information}

JAS has served as the cochair of the gout working group of the Outcome Measures in Rheumatology (OMERACT) initiative.

\section{Ethics approval and consent to participate}

The institutional review board (IRB) at the University of Alabama at Birmingham approved this study, and all investigations were conducted in conformity with ethical principles of research. The IRB waived the need for written informed consent for this retrospective database study.

\section{Consent for publication}

\section{Not applicable.}

\section{Competing interests}

JAS has received research grants from Takeda Pharmaceuticals and Savient Pharmaceuticals and consultant fees from Savient Pharmaceuticals, Takeda Pharmaceuticals, Regeneron Pharmaceuticals, Merz, Bioibérica, Crealta Pharmaceuticals, Allergan, WebMD, UBM LLC, and the American College of Rheumatology (ACR). JAS serves as the principal investigator for an investigator-initiated study funded by Horizon Pharma through a grant to DINORA, Inc., a 501 (c)(3) entity. JAS is a member of the executive committee of OMERACT, an organization that develops outcome measures in rheumatology and receives arm's-length funding from 36 companies; a member of the ACR Annual Meeting Planning Committee; chair of the ACR Meet the Professor, Workshop, and Study Group Subcommittee; and a member of the Veterans Affairs Rheumatology Field Advisory Committee. JRC has received research grant funding via the University of Alabama at Birmingham and consulting monies for unrelated work from AbbVie, Amgen, Bristol-Myers Squibb, Janssen, Pfizer, Roche, and UCB. The other authors declare that they have no competing interests. None of the authors have any other nonfinancial disclosures.

\section{Publisher's Note}

Springer Nature remains neutral with regard to jurisdictional claims in published maps and institutional affiliations.

\section{Author details}

'Medicine Service, VA Medical Center, 700 South 19th Street, Birmingham, AL 35233, USA. ${ }^{2}$ Department of Medicine, University of Alabama at Birmingham School of Medicine, 510 20th Street South, Faculty Office Tower 805B, Birmingham, AL 35294-0022, USA. ${ }^{3}$ Division of Epidemiology, University of Alabama at Birmingham School of Public Health, 1720 Second Avenue South, Birmingham, AL 35294-0022, USA. ${ }^{4}$ University of Alabama at Birmingham, 510 20th Street South, Faculty Office Tower 805B, Birmingham, AL 35294, USA.

\section{Received: 26 January 2017 Accepted: 11 September 2017}

\section{Published online: 17 October 2017}

\section{References}

1. Zhu Y, Pandya BJ, Choi HK. Prevalence of gout and hyperuricemia in the US general population: the National Health and Nutrition Examination Survey 2007-2008. Arthritis Rheum. 2011;63(10):3136-41.

2. Abbott RD, Brand FN, Kannel WB, Castelli WP. Gout and coronary heart disease: the Framingham Study. J Clin Epidemiol. 1988;41(3):237-42.

3. Choi HK, Curhan G. Independent impact of gout on mortality and risk for coronary heart disease. Circulation. 2007;116(8):894-900.

4. Cohen SD, Kimmel PL, Neff R, Agodoa L, Abbott KC. Association of incident gout and mortality in dialysis patients. J Am Soc Nephrol. 2008;19(11):2204-10.

5. De Vera MA, Rahman MM, Bhole V, Kopec JA, Choi HK. Independent impact of gout on the risk of acute myocardial infarction among elderly women: a population-based study. Ann Rheum Dis. 2010;69(6):1162-4.

6. Krishnan E, Baker JF, Furst DE, Schumacher HR. Gout and the risk of acute myocardial infarction. Arthritis Rheum. 2006;54(8):2688-96.

7. Seminog $\mathrm{OO}$, Goldacre MJ. Gout as a risk factor for myocardial infarction and stroke in England: evidence from record linkage studies. Rheumatology (Oxford). 2013;52(12):2251-9.

8. Kuo CF, Yu KH, See LC, Chou IJ, Ko YS, Chang HC, Chiou MJ, Luo SF. Risk of myocardial infarction among patients with gout: a nationwide populationbased study. Rheumatology (Oxford). 2013;52(1):111-7.
9. Gelber AC, Klag MJ, Mead LA, Thomas J, Thomas DJ, Pearson TA, Hochberg MC. Gout and risk for subsequent coronary heart disease: the MeharryHopkins Study. Arch Intern Med. 1997;157(13):1436-40.

10. Janssens HJ, van de Lisdonk EH, Bor H, van den Hoogen HJ, Janssen M. Gout, just a nasty event or a cardiovascular signal? A study from primary care. Fam Pract. 2003;20(4):413-6.

11. Kim SY, Guevara JP, Kim KM, Choi HK, Heitjan DF, Albert DA. Hyperuricemia and risk of stroke: a systematic review and meta-analysis. Arthritis Rheum. 2009;61(7):885-92

12. Kim SY, Guevara JP, Kim KM, Choi HK, Heitjan DF, Albert DA. Hyperuricemia and coronary heart disease: a systematic review and meta-analysis. Arthritis Care Res (Hoboken). 2010;62(2):170-80.

13. Johnson RJ, Kang DH, Feig D, Kivlighn S, Kanellis J, Watanabe S, Tuttle KR, Rodriguez-Iturbe B, Herrera-Acosta J, Mazzali M. Is there a pathogenetic role for uric acid in hypertension and cardiovascular and renal disease? Hypertension. 2003;41(6):1183-90.

14. Feig DI, Kang DH, Johnson RJ. Uric acid and cardiovascular risk. N Engl J Med. 2008;359(17):1811-21.

15. National Cholesterol Education Program (NCEP) Expert Panel on Detection, Evaluation, and Treatment of High Blood Cholesterol in Adults (Adult Treatment Panel III). Third Report of the National Cholesterol Education Program (NCEP) Expert Panel on Detection, Evaluation, and Treatment of High Blood Cholesterol in Adults (Adult Treatment Panel III) final report. Circulation. 2002;106(25):3143-421.

16. Singh JA. When gout goes to the heart: does gout equal a cardiovascular disease risk factor? Ann Rheum Dis. 2015;74(4):631-4.

17. STrengthening the Reporting of OBservational studies in Epidemiology (STROBE) Initiative. STROBE Statement—checklist of items that should be included in reports of cohort studies. http://www.strobe-statement.org/ fileadmin/Strobe/uploads/checklists/STROBE_checklist_v4_cohort.pdf. Accessed 20 Sept 2017.

18. Singh JA, Hodges JS, Toscano JP, Asch SM. Quality of care for gout in the US needs improvement. Arthritis Rheum. 2007;57(5):822-9.

19. Miller DR, Safford MM, Pogach LM. Who has diabetes? Best estimates of diabetes prevalence in the Department of Veterans Affairs based on computerized patient data. Diabetes Care. 2004;27 Suppl 2:B10-21.

20. Singh JA. Accuracy of Veterans Affairs databases for diagnoses of chronic diseases. Prev Chronic Dis. 2009;6(4):A126.

21. Solberg LI, Engebretson Kl, Sperl-Hillen JM, Hroscikoski MC, O'Connor PJ. Are claims data accurate enough to identify patients for performance measures or quality improvement? The case of diabetes, heart disease, and depression. Am J Med Qual. 2006;21(4):238-45.

22. Birman-Deych E, Waterman AD, Yan Y, Nilasena DS, Radford MJ, Gage BF. Accuracy of ICD-9-CM codes for identifying cardiovascular and stroke risk factors. Med Care. 2005;43(5):480-5.

23. Kiyota Y, Schneeweiss S, Glynn RJ, Cannuscio CC, Avorn J, Solomon DH. Accuracy of Medicare claims-based diagnosis of acute myocardial infarction: estimating positive predictive value on the basis of review of hospital records. Am Heart J. 2004;148(1):99-104.

24. Thigpen JL, Dillon C, Forster KB, Henault L, Quinn EK, Tripodis Y, Berger PB, Hylek EM, Limdi NA. Validity of international classification of disease codes to identify ischemic stroke and intracranial hemorrhage among individuals with associated diagnosis of atrial fibrillation. Circ Cardiovasc Qual Outcomes. 2015;8(1):8-14

25. Jollis JG, Ancukiewicz M, DeLong ER, Pryor DB, Muhlbaier LH, Mark DB. Discordance of databases designed for claims payment versus clinical information systems: implications for outcomes research. Ann Intern Med. 1993;119(8):844-50.

26. Andres M, Bernal JA, Sivera F, Quilis N, Carmona L, Vela P, et al. Cardiovascular risk of patients with gout seen at rheumatology clinics following a structured assessment. Ann Rheum Dis 2017;76:1263-8.

27. Choi YJ, Yoon Y, Lee KY, Hien TT, Kang KW, Kim KC, Lee J, Lee MY, Lee SM, Kang $\mathrm{DH}$, et al. Uric acid induces endothelial dysfunction by vascular insulin resistance associated with the impairment of nitric oxide synthesis. FASEB J. 2014;28(7):3197-204.

28. Gersch C, Palii SP, Kim KM, Angerhofer A, Johnson RJ, Henderson GN. Inactivation of nitric oxide by uric acid. Nucleosides Nucleotides Nucleic Acids. 2008;27(8):967-78.

29. Yu MA, Sanchez-Lozada LG, Johnson RJ, Kang DH. Oxidative stress with an activation of the renin-angiotensin system in human vascular endothelial cells as a novel mechanism of uric acid-induced endothelial dysfunction. J Hypertens. 2010;28(6):1234-42. 
30. Kanellis J, Kang DH. Uric acid as a mediator of endothelial dysfunction, inflammation, and vascular disease. Semin Nephrol. 2005;25(1):39-42.

31. Sanchez-Lozada LG, Lanaspa MA, Cristobal-Garcia M, Garcia-Arroyo F, Soto V, Cruz-Robles D, Nakagawa T, Yu MA, Kang DH, Johnson RJ. Uric acid-induced endothelial dysfunction is associated with mitochondrial alterations and decreased intracellular ATP concentrations. Nephron Exp Nephrol. 2012;121(3-4):e71-8.

32. Feig DI, Nakagawa T, Karumanchi SA, Oliver WJ, Kang DH, Finch J, Johnson RJ. Hypothesis: uric acid, nephron number, and the pathogenesis of essential hypertension. Kidney Int. 2004;66(1):281-7.

33. Mazzali M, Hughes J, Kim YG, Jefferson JA, Kang DH, Gordon KL, Lan HY, Kivlighn S, Johnson RJ. Elevated uric acid increases blood pressure in the rat by a novel crystal-independent mechanism. Hypertension. 2001;38(5):1101-6.

34. Kanellis J, Watanabe S, Li JH, Kang DH, Li P, Nakagawa T, Wamsley A, SheikhHamad D, Lan HY, Feng L, et al. Uric acid stimulates monocyte chemoattractant protein-1 production in vascular smooth muscle cells via mitogen-activated protein kinase and cyclooxygenase-2. Hypertension. 2003;41(6):1287-93.

35. Valle M, Martos R, Cañete MD, Valle R, van Donkelaar EL, Bermudo F, Cañete R Association of serum uric acid levels to inflammation biomarkers and endothelial dysfunction in obese prepubertal children. Pediatr Diabetes. 2015;16(6):441-7.

36. Buckland J. Crystal arthropathies: the NET is closing in on inflammation in gout. Nat Rev Rheumatol. 2014;10(6):319.

37. Dalbeth $\mathrm{N}$, Haskard DO. Mechanisms of inflammation in gout. Rheumatology (Oxford). 2005;44(9):1090-6.

38. Tiong AY, Brieger D. Inflammation and coronary artery disease. Am Heart J. 2005;150(1):11-8.

39. Rao GN, Corson MA, Berk BC. Uric acid stimulates vascular smooth muscle cell proliferation by increasing platelet-derived growth factor A-chain expression. J Biol Chem. 1991;266(13):8604-8.

40. Corry DB, Eslami P, Yamamoto K, Nyby MD, Makino H, Tuck ML. Uric acid stimulates vascular smooth muscle cell proliferation and oxidative stress via the vascular renin-angiotensin system. J Hypertens. 2008;26(2):269-75.

41. Chao HH, Liu JC, Lin JW, Chen CH, Wu CH, Cheng TH. Uric acid stimulates endothelin-1 gene expression associated with NADPH oxidase in human aortic smooth muscle cells. Acta Pharmacol Sin. 2008;29(11):1301-12.

42. Gur M, Sahin DY, Elbasan Z, Kalkan GY, Yildiz A, Kaya Z, Ozaltun B, Cayli M. Uric acid and high sensitive C-reactive protein are associated with subclinical thoracic aortic atherosclerosis. J Cardiol. 2013:61(2):144-8.

43. Neogi T, Ellison RC, Hunt S, Terkeltaub R, Felson DT, Zhang Y. Serum uric acid is associated with carotid plaques: the National Heart, Lung, and Blood Institute Family Heart Study. J Rheumatol. 2009;36(2):378-84.

44. Kuo CF, Grainge MJ, Mallen C, Zhang W, Doherty M. Impact of gout on the risk of atrial fibrillation. Rheumatology (Oxford). 2016;55(4):721-8.

45. Kim SC, Liu J, Solomon DH. Risk of incident atrial fibrillation in gout: a cohort study. Ann Rheum Dis. 2016;75(8):1473-8.

46. Ivanusa M, Ivanusa Z. Risk factors and in-hospital outcomes in stroke and myocardial infarction patients. BMC Public Health. 2004;4:26.

47. Kennedy RE, Howard G, Go RC, Rothwell PM, Tiwari HK, Feng R, McClure LA, Prineas RJ, Banerjee A, Arnett DK. Association between family risk of stroke and myocardial infarction with prevalent risk factors and coexisting diseases. Stroke. 2012:43(4):974-9.

48. Uchiyama S, Shibata Y, Hirabayashi T, Mihara B, Hamashige N, Kitagawa K, Goto S, Origasa H, Shimada K, Kobayashi H, et al. Risk factor profiles of stroke, myocardial infarction, and atrial fibrillation: a Japanese Multicenter Cooperative Registry. J Stroke Cerebrovasc Dis. 2010;19(3):190-7.

49. Kuo CF, See LC, Luo SF, Ko YS, Lin YS, Hwang JS, Lin CM, Chen HW, Yu KH. Gout: an independent risk factor for all-cause and cardiovascular mortality. Rheumatology (Oxford). 2010;49(1):141-6.

50. Krishnan E, Svendsen K, Neaton JD, Grandits G, Kuller LH. Long-term cardiovascular mortality among middle-aged men with gout. Arch Intern Med. 2008;168(10):1104-10

51. Biscaglia S, Ceconi C, Malagu M, Pavasini R, Ferrari R. Uric acid and coronary arten disease: an elusive link deserving further attention. Int J Cardiol. 2016;213:28-32.

52. Jacobs MJ, Kleisli T, Pio JR, Malik S, L'Italien GJ, Chen RS, Wong ND. Prevalence and control of dyslipidemia among persons with diabetes in the United States. Diabetes Res Clin Pract. 2005;70(3):263-9.

53. McDonald M, Hertz RP, Unger AN, Lustik MB. Prevalence, awareness, and management of hypertension, dyslipidemia, and diabetes among United States adults aged 65 and older. J Gerontol A Biol Sci Med Sci. 2009;64(2):256-63

\section{Submit your next manuscript to BioMed Central and we will help you at every step:}

- We accept pre-submission inquiries

- Our selector tool helps you to find the most relevant journal

- We provide round the clock customer support

- Convenient online submission

- Thorough peer review

- Inclusion in PubMed and all major indexing services

- Maximum visibility for your research

Submit your manuscript at www.biomedcentral.com/submit
) Biomed Central 